\title{
A rapid desensitization protocol in a case of drotaverine-induced serum sickness-like reaction in a pregnant woman: A case report
}

\author{
SELDA ALI $^{1,2}$, SABINA LOREDANA CORCEA $^{3}$, RODICA MARIA CRISTIAN $^{4}$ and ROXANA SILVIA BUMBACEA ${ }^{1,2}$ \\ ${ }^{1}$ Department of Allergy, 'Dr. Carol Davila' Nephrology Clinical Hospital, 010731 Bucharest; \\ Departments of ${ }^{2}$ Allergy, and ${ }^{3}$ Physiopathology and Immunology, 'Carol Davila' University of Medicine and Pharmacy, \\ 050474 Bucharest; ${ }^{4}$ Department of Obstetrics and Gynecology, Medlife Clinic, 013682 Bucharest, Romania
}

Received September 6, 2019; Accepted October 7, 2019

DOI: $10.3892 /$ etm.2019.8170

\begin{abstract}
Drotaverine is an antispasmodic drug used to treat gastrointestinal and genitourinary smooth muscle spasms. There are very few hypersensitivity reactions reported. Serum sickness-like disease is an immune-complex-mediated hypersensitivity reaction that presents with some typical features that include rash, fever and articular impairment sometimes associated with liver and renal dysfunctions, beginning 1-2 weeks after exposure to a culprit drug. Diagnosis is a clinical one, made usually on the basis of knowledge obtained by medical history and physical examination. Desensitization usually is recommended for type I reaction, but may be a solution for this type of immunological reaction when other therapeutic alternatives are ineffective or do not exist. We report the case of a 29-year-old pregnant female who developed serum sickness-like reaction after 5 days of daily drotaverine oral administration. The patient required antispasmodic treatment, with this drug, having a pregnancy with an imminent risk of abortion and the other therapeutic alternatives being ineffective. She underwent a rapid 7-step oral drotaverine desensitization protocol without recurrence of serum sickness-like reaction. To our knowledge, this is the first case report of desensitization to drotaverine, previously involved in a serum sickness-like reaction.
\end{abstract}

\section{Introduction}

Serum sickness disease (SSD) represents the common example for Gell and Coombs 'type III' reaction, an immune-complex mediated hypersensitivity reaction (1). SSD is caused by the

Correspondence to: Dr Selda Ali, Department of Allergy, 'Dr. Carol Davila' Nephrology Clinical Hospital, 4 Grivita Street, 010731 Bucharest, Romania

E-mail: aliselda.ct@gmail.com

Abbreviations: SSD, serum sickness disease

Keywords: drotaverine, serum sickness, immune-complex-mediated hypersensitivity reaction, pregnancy, desensitization protocol administration of heterologous antisera. SSD-like reactions have been described after exposure to other drugs, especially cefaclor and anti-seizure medications. Nowadays SSD and SSD-like reactions are rare entities, SSD being more common in adults and SSD-like reactions being more common in children (2).

Serum sickness-like reaction consists of typical clinical features (rash, malaise, polyarthralgia and polyarthritis, sometimes associated with lymphadenopathy, liver and renal dysfunctions) that begin one to two weeks after exposure to the causing drug or even earlier in a patient who has been previously exposed to the same agent (2).

Diagnosis is usually made by medical history and physical examination, after excluding alternative diagnoses. There is no specific laboratory finding, but erythrocyte sedimentation rate may be increased, accompanied by leucopenia or leucocytosis, hypocomplementemia (C3 and C4 fractions) and proteinuria.

\section{Case report}

A 29-year-old female with an 8-week ongoing pregnancy (4th pregnancy) who developed symptoms compatible with drotaverine-induced serum sickness disease and who needed antispasmodic treatment was referred to our drug allergy department in order to perform an allergy work-up and make a therapeutic decision.

Four weeks prior to admission, the patient was administered drotaverine $40 \mathrm{mg}$ (No-Spa) three times a day and after approximately 5 days, she started to have malaise, pain, swelling and mild stiffness of the elbows, knees and metacarpophalangeal joints associated with a diffuse rash, mostly in the lower limbs. During last three pregnancies the patient used drotaverine frequently without any adverse reactions. The patient did not complain of fever or other systemic symptoms, but the articular symptoms were intense and significantly altered her quality of life.

She was evaluated by a primary care physician who administered $200 \mathrm{mg}$ of hydrocortisone sodium succinate with rapid resolution of the cutaneous symptoms.

During the following two days, the articular symptomatology persisted and our allergy evaluation was requested; the diagnosis of serum sickness-like reaction was suggested 
Table I. Desensitization schedule.

\begin{tabular}{|c|c|c|c|}
\hline Day & Number of dosages & Dose (mg) & Time $(\mathrm{min})$ \\
\hline \multirow[t]{7}{*}{1} & 1 & 1 & 30 \\
\hline & 2 & 2 & 30 \\
\hline & 3 & 3 & 30 \\
\hline & 4 & 8 & 30 \\
\hline & 5 & 16 & 30 \\
\hline & 6 & 32 & 30 \\
\hline & 7 & 40 & 30 \\
\hline \multirow[t]{3}{*}{2} & 1 & 40 & 360 \\
\hline & 2 & 40 & 360 \\
\hline & 3 & 40 & 360 \\
\hline \multirow[t]{3}{*}{3} & 1 & 40 & 360 \\
\hline & 2 & 40 & 360 \\
\hline & 3 & 40 & 360 \\
\hline
\end{tabular}

and consecutively extensive laboratory investigations were performed. Complete blood count analysis, inflammatory biomarkers, renal and hepatic functions, C3 and C4 complement fractions, serologies for rheumatoid factor, cyclic citrullinated peptide, HLA B27, antinuclear antibodies, urine analysis were within normal ranges with the exception of mild inflammatory syndrome (erythrocyte sedimentation rate $-34 \mathrm{~mm} / \mathrm{h}$ (normal ranges: $4-20 \mathrm{~mm} / \mathrm{h}$ ) and C3 - $40 \mathrm{mg} / \mathrm{dl}$ (normal ranges: $90-180 \mathrm{mg} / \mathrm{dl}$ ). Screening for viral arthritis (hepatitis B, C, EBV and rubella) was also negative.

Given the patient's symptoms of rash, malaise and polyarthralgia and polyarthritis, a consistent time course with signs and symptoms arising within 5 days following the start of treatment, and negative work-up for alternative diagnoses, a diagnosis of SSD-like reaction was made.

We decided to stop the offending drug and to prescribe systemic corticosteroid (a short schedule of prednisone with a starting dose of $25 \mathrm{mg}$ per day that was progressively decreased for 7 days). The resolution of articular symptoms was very rapid ( 3 days after starting the treatment).

Later on, obstetrical evaluation decided that the administration of drotaverine is indispensable and she was referred to our clinic as in-patient for allergy work-up. At admission, physical examination did not reveal abnormalities. Laboratory work-up for complete blood count, inflammatory biomarkers (CRP, fibrinogen and ESR), renal function tests (serum creatinine, uric acid, blood urea nitrogen), liver function tests (aspartate aminotransferase and alanine aminotransferase), total proteins and albumin, C3 and C4 complement fractions were within normal ranges. Routine urine examination was normal and culture showed no infection. Serum IgE level was $23.90 \mathrm{IU} / \mathrm{ml}$. Atopic status was not confirmed after skin prick tests to European skin prick test panel for respiratory allergens.

Given the need for antispasmodic treatment (abortion risk) and the lack of response to other antispasmodic alternatives we designed a desensitization schedule (Table I) with a 3-day pre-treatment regimen with low dose of oral corticosteroids (prednisone-10 mg/day). We obtained the informed consent of the patient.
After the desensitization protocol, the patient continued the treatment and tolerated the intake of drotaverine 1 tablet $(40 \mathrm{mg})$ three times daily. At the time of this case report, she remains free of symptoms.

To our knowledge, this is the first report of successful drug desensitization to drotaverine in a pregnant patient with drotaverine-induced serum sickness disease-like reaction.

The study was approved by the Ethics Committee of 'Dr. Carol Davila' Nephrology Clinical Hospital (Bucharest, Romania), and signed informed consent was obtained from the patient in the study.

\section{Discussion}

SSD-like reaction is a rare condition. The pathogenesis is poorly understood; it is generally believed that it is an inflammatory response to drug metabolites. The diagnosis is usually based on clinical symptoms and, if present, on laboratory findings. Desensitization protocols are available for immediate-type reactions, but for delayed type drug induced hypersensitivity reactions there are only case reports or case series (3). There is no consensus on the number of steps for reaching the recommended dose or for the usefulness of premedication (4).

In Romania, drotaverine is commonly used in pregnant patients with abortion risk. It is an 'off-label' prescription because the summary of product characteristics states 'As a precaution, it is preferable to avoid using No-Spa during pregnancy.' No-spa is the commercial name of drotaverine in Romania. Drotaverine is considered to be 'unclassified medication' used during pregnancy depending on its safety profile according to Tronnes et al (5). However, a newer study considers it a 'probably safe' drug to be used during pregnancy and states that it is in the top drugs used during pregnancy (6).

In female patients with high risk pregnancies there are few drugs available. Our patient had no other drug option available. For IgE-mediated reactions skin prick tests or intradermal tests are used for confirming the diagnosis, but for serum sickness-like reactions there are no available tests. Considering our patient was pregnant we did not perform a drug provocation test (7).

We designed a 7-step protocol for desensitization to drotaverine. We used low doses of systemic corticosteroids as premedication in order to induce tolerance. The reason for using a low dose corticosteroid was the pregnancy (7).

Desensitization in serum sickness type disease may be possible and may enable patients to continue an effective and safe treatment. Depending on the type and the onset of the hypersensitivity reaction, the physician may modify the protocol. This kind of procedures must be performed only by doctors experienced in drug desensitization. The patient must be closely monitored and immediate emergency treatment must be available. Even after reaching the recommended dose and discharging, the patient has to be able to recognize the early signs of a hypersensitivity reaction and address for evaluation and treatment (8).

The tolerance obtained through desensitization usually persists as long as the drug therapy is continued without interruption. If the patient needs the same drug again after 
discontinuing the treatment, another desensitization protocol must be performed. In our patient, drotaverine will be daily administered until the moment of childbirth. If the patient would not complete the desensitization procedure, another option for inducing tolerance could have been pre-treatment with omalizumab. Omalizumab is currently also used for the treatment of chronic severe urticaria (9). It has also been labelled as a safe drug to be used during pregnancy with no increased risk of major abnormalities between foetuses whose mothers were on treatment with omalizumab and the control group (10).

The particularity of the case is that our patient developed SSD after 5 days of spasmolytic treatment with drotaverine, earlier than usual, probably because of the frequent previous administration of the drug.

\section{Acknowledgements}

Not applicable.

\section{Funding}

No funding was received.

\section{Availability of data and materials}

The datasets used and/or analyzed during the current study are available from the corresponding author on reasonable request.

\section{Authors' contributions}

SA, RSB and SLC contributed to the acquisition of the data, the conception and the design of the study. RMC is the gynecologist of the patient, who provided data about the usefulness of drotaverine during high risk pregnancies and contributed to the analysis of data for the study. All authors were responsible for drafting and revising the manuscript. All authors read and approved the final manuscript.

\section{Ethics approval and consent to participate}

The study was approved by the Ethics Committee of 'Dr. Carol Davila' Nephrology Clinical Hospital (Bucharest, Romania), and signed informed consent was obtained from the patient in the study.

\section{Patient consent for publication}

Signed informed consent was obtained from the patient in the study.

\section{Competing interests}

The authors declare that they have no conflicts of interest.

\section{References}

1. Lawley TJ, Bielory L, Gascon P, Yancey KB, Young NS and Frank MM: A prospective clinical and immunologic analysis of patients with serum sickness. N Engl J Med 311: 1407-1413, 1984.

2. Wener MH: Serum sickness and serum sickness-like reaction, UpToDate, 2019. https://www.uptodate.com/contents/serumsickness-and-serum-sickness-like-reactions. Accessed June 30, 2019.

3. Cernadas JR, Brockow K, Romano A, Aberer W, Torres MJ, Bircher A, Campi P, Sanz ML, Castells M, Demoly P, et al; European Network of Drug Allergy and the EAACI interest group on drug hypersensitivity: General considerations on rapid desensitization for drug hypersensitivity - a consensus statement. Allergy 65: 1357-1366, 2010.

4. Scherer K, Brockow K, Aberer W, Gooi JHC, Demoly P, Romano A, Schnyder B, Whitaker P, Cernadas JSR and Bircher AJ; ENDA, the European Network on Drug Allergy and the EAACI Drug Allergy Interest Group: Desensitization in delayed drug hypersensitivity reactions - an EAACI position paper of the Drug Allergy Interest Group. Allergy 68: 844-852, 2013.

5. Trønnes JN, Lupattelli A and Nordeng H: Safety profile of medication used during pregnancy: results of a multinational European study. Pharmacoepidemiol Drug Saf 26: 802-811, 2017.

6. Rouamba T, Valea I, Bognini JD, Kpoda H, Mens PF, Gomes MF, Tinto H, Kirakoya-Samadoulougou F: Safety profile of drug use during pregnancy at peripheral health centres in burkina faso: a prospective observational cohort study. Drugs Real World Outcomes 5: 193-206, 2018

7. Crowther CA, Middleton PF, Voysey M, Askie L, Zhang S, Martlow TK, Aghajafari F, Asztalos EV, Brocklehurst P, Dutta S, et al; PRECISE Group: Effects of repeat prenatal corticosteroids given to women at risk of preterm birth: An individual participant data meta-analysis. PLoS Med 16: e1002771, 2019.

8. Cernadas JR, Brockow K, Romano A, Aberer W, Torres MJ, Bircher A, Campi P, Sanz ML, Castells M, Demoly P, Pichler WJ; European Network of Drug Allergy and the EAACI interest group on drug hypersensitivity: General considerations on rapid desensitization for drug hypersensitivity - a consensus statement. Allergy 65: 1357-1366, 2010.

9. Bumbăcea RS, Deaconu CG and Berghea EC: Management problems in severe chronic inducible urticaria: Two case reports. Exp Ther Med 18: 960-963, 2019.

10. Namazy JA, Blais L, Andrews EB, Scheuerle AE, Cabana MD, Thorp JM, Umetsu DT, Veith JH, Sun D, Kaufman DG, et al: Pregnancy outcomes in the omalizumab pregnancy registry and a disease-matched comparator cohort. J Allergy Clin Immunol. May 27, 2019 (Epub ahead of print). 